\title{
Adaptive Channel Allocation in OFDM/SDMA Wireless LANs with Limited Transceiver Resources
}

\author{
Iordanis Koutsopoulos and Leandros Tassiulas \\ Department of Computer and Communications Engineering, \\ University of Thessaly, Greece
}

\begin{abstract}
Smart antennas increase capacity of wireless systems by allowing channel reuse by several users in the same cell through space division multiple access (SDMA). Orthogonal Frequency Division Multiplexing (OFDM) creates additional challenges in beam-forming when the beams that can be formed are limited due to transceiver unit limitations. We investigate the impact of smart antennas on channel allocation and propose meaningful heuristics to form beams and assign subcarriers and transceivers to users. Numerical results quantify performance benefits and provide useful design guidelines.
\end{abstract}

\section{Introduction}

A smart antenna enables intra-cell channel reuse by many spatially separable users by steering the main lobe of each beam to the desired direction and placing nulls in the directions of interfering users [1]. Smart antennas at the physical layer raise significant issues at higher layers that have not been addressed in literature. For instance, channel allocation has hitherto been studied separately from spatial separation and channel reuse. The latter are based on static cell sectorization or beam switching methods that do not capture channel dynamics, mobility and traffic load variations. Orthogonal frequency division multiplexing (OFDM) is a signaling and access technique for wireless broadband networks that provides immunity to inter-symbol interference (ISI) and high data rates [2]. The spectrum is divided into orthogonal narrow-band subcarriers and the user bit stream is split into subsymbols that are transmitted in parallel over subcarriers.

An unaddressed issue concerns transceiver limitations that are significant in indoor WLANs or WPANs, where implementation complexity and cost, physical space inadequacy or specifications on maximum induced interference may limit the number of beams that can be formed. A first category of studies consider single-channel multi-user systems [3], [4]. For $M$ antennas, $M$ transceiver modules are required to form a beam for each user in the co-channel set. This also holds for multi-channel multi-user systems with time division multiplexing [5], where co-channel sets change in different slots. In OFDM, each of the $N$ subcarriers has different quality for a user due to different impact of frequency on user 


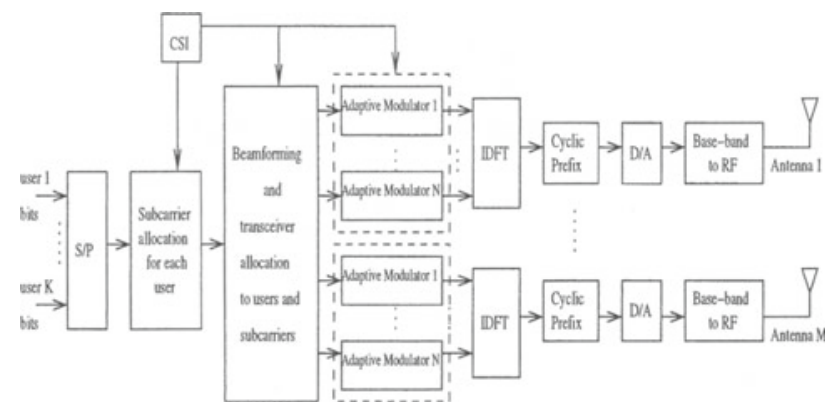

Fig. 1. Multi-user OFDM/SDMA transmitter with limited transceiver resources.

spatial and multi-path characteristics. In single-user systems, $N$ transceivers are required [6]. However, in a multi-user OFDM system, a separate beam may be needed for each user in a co-channel set in each subcarrier and $N M$ transceivers may be needed. Depending on $N$ and $M$, this number can be of the order of several hundreds and may not comply with the limitations above. In [7], the existence of $N M$ transceivers was implied. We investigate the impact of smart antenna systems with limited transceiver resources on channel allocation. This paper provides a brief overview of the topic. For a more detailed study, the reader is referred to [8].

We consider OFDM transmission with $N$ subcarriers from an access point (AP) with a smart antenna of $M$ elements to $K$ users. The block diagram is depicted in figure 1. Each of the $C$ transceivers can form a unit-power beam $\mathbf{u}_{c}=\left(u_{c}^{1}, \ldots, u_{c}^{M}\right)^{T}$. The expected SIR at the receiver of user $k$ at subcarrier $n$ that receives useful signal from transceiver $c$ is $\operatorname{SINR}_{n, k}^{c}=$ $\left(\mathbf{u}_{c}^{H} \mathcal{H}_{n, k} \mathbf{u}_{c}\right) /\left(\sum_{b=1, b \neq c}^{C} \mathbf{u}_{b}^{H} \mathcal{H}_{n, k} \mathbf{u}_{b}\right)$, where $\mathcal{H}_{n, k}$ is called spatial covariance matrix of user $k$ and captures angular and multi-path characteristics such as path gains and delays and their impact on subcarrier $n$. Matrices $\mathcal{H}_{n, k}$ can be estimated by sending known pilot symbols in the up-link. The BER at the output of the detector in a subcarrier must satisfy $\mathrm{BER} \leq \epsilon$. For one modulation level with $b_{0}$ bits/subsymbol, the minimum required SIR (in $\mathrm{dB}$ ) for this BER specification is $\gamma=-(\ln (5 \epsilon) / 1.5)\left(2^{b_{0}}-1\right)$.

\section{Channel Allocation for OFDM/SDMA Systems with Limited Transceiver Resources}

Each user receives useful signal from a transceiver and co-channel interference from beams of other transceivers that use the same subcarrier for other users. The co-channel set is spatially separable if there exist beams, one from each transceiver, so that a minimum SIR is ensured for each user. Spatial separability depends on user spatial covariance matrices, on the specific subcarrier and on beam-forming vectors. Clearly, users that are illuminated by one beam must use 
different subcarriers. Users that are served by different beams may or may not reuse a subcarrier, depending on user spatial and multi-path characteristics at the subcarrier, beam orientations and induced co-channel interference by beams. A user experiences co-channel interference from beams of other transceivers that use the same subcarrier. Therefore, subcarrier and transceiver assignment are coupled. Our algorithms consist of two stages. First, users are assigned to subcarriers and beams are computed assuming there are no transceiver limitations. In the second stage, the beams are sequentially unified, until the desired number of $C$ beams is reached.

In the first stage, the idea is to create large co-channel sets of spatially separable users in each subcarrier. An appropriate user is sequentially assigned to a subcarrier and beams of co-channel users are adjusted so that acceptable SIRs are ensured. Inserted users should cause least interference to users that are already assigned in the channel and should receive least interference from them. For each inserted user $k$, we evaluate a factor

$$
\Phi_{n, k}=\frac{\mathbf{u}_{n, k}^{* H} \mathcal{H}_{n, k} \mathbf{u}_{n, k}^{*}}{\max \left\{\mathbf{u}_{n, k}^{* H}\left(\sum_{j \in \mathcal{U}^{(n)}} \mathcal{H}_{n, j}\right) \mathbf{u}_{n, k}^{*}, \sum_{j \in \mathcal{U}^{(n)}} \mathbf{u}_{n, j}^{* H} \mathcal{H}_{n, k} \mathbf{u}_{n, j}^{*}\right\}},
$$

where beams maximize the ratio of created useful and interference signals. This factor captures the requirement of high useful signal power and least caused and received interference to or from other users in $n$. The assignment of a user with the maximum $\Phi_{n, k}$ is desired. Users are sequentially inserted in each subcarrier until one SIR is violated and this is performed for all subcarriers.

In the second stage, we need to reduce the number of beams to $C$ while maintaining high subcarruer reuse. Clearly, only beams from different subcarriers can be unified to a new beam, since the new beam cannot serve users of the same subcarrier. At each iteration of the unification algorithm, we need to select an appropriate beam pair from different subcarriers $n, m$ and replace it with a new beam. The rationale for selecting the beam pair is to combine beams of different subcarriers with similar orientations, so that desirable properties of old beams are maintained. Thue, the beam pair with minimum Euclidean distance is selected. For normalized beams, this is equivalent to $\left(k^{*}, \ell^{*}\right)=\arg \max _{(k, \ell)} \Re\left(\rho_{k \ell}\right)$, where $\rho_{k, \ell}$ is the beam cross-correlation. The new beam $\mathbf{u}_{c}$ that replaces old beams $\mathbf{u}_{n, k}$ and $\mathbf{u}_{m, \ell}$ can be computed with one of the following methods.

Method A: Maximum new/old beam cross-correlation. New beam $\mathbf{u}_{c}^{*}$ should have maximum cross-correlation with old beams, subject to being normalized and is thus the solution of a constrained optimization problem. After computing $\mathbf{u}_{c}^{*}$, we replace $\mathbf{u}_{n, k}$ and $\mathbf{u}_{m, \ell}$ with $\mathbf{u}_{c}^{*}$ and evaluate user SIRs in $k, \ell$ and SIRs of other users in old beams which are affected by the replacement. If all SIRs exceed $\gamma$, we replace beams $\mathbf{u}_{n, k}$ and $\mathbf{u}_{m, \ell}$ with beam $\mathbf{u}_{c}^{*}$ and proceed to selecting the next beam pair. Otherwise, some beams (and thus users served by these beams) in subcarriers $n$ and $m$ must be removed, so that SIRs increase. We eliminate the user that maximizes the minimum SIR, since we intend to maintain a large number of users with acceptable SIRs. This continues until SIRs of all users are 
acceptable. Then, the algorithm proceeds to selecting the next beam pair and the procedure terminates when the number of beams is $C$.

Method B: Maximum signal strength/minimum induced interference. The new beam $\mathbf{u}_{c}^{*}$ must maximize the ratio of total useful power for users $k$ and $\ell$ in initial beams and total interference to other users. After computing $\mathbf{u}_{c}^{*}$, SIRs of users are calculated and users are sequentially eliminated, until acceptable SIRs are ensured.

Note that as the algorithm progresses, one or both beams that are unified may contain several users in different subcarriers or a user with several subcarriers. All such users are included in the algorithm.

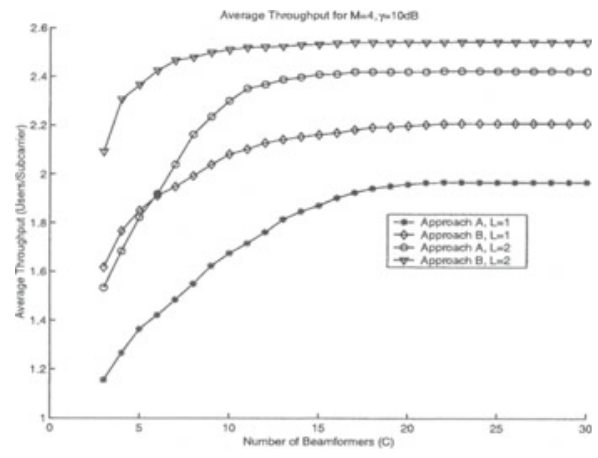

Fig. 2. Average throughput vs. number of transceivers for approaches $A$ and $B$, for multi-path with $L=1$ and $L=2$ paths and $M=4$ antennas.

\section{Simulation Results}

We simulate a system with an $\mathrm{AP}, N=10$ subcarriers and $K=15$ users. In both of the considered approaches, the first stage is executed and the beam pairs for unification are selected with the cross-correlation criterion. The differences of the approaches are as follows. In approach A, the new beam is computed with method A. Next, beams are sequentially eliminated, until SIRs of remaining users exceed $\gamma$. In B, the new beam is computed with method B. Now, after beam elimination, a new beam is computed again with a ratio similar to that in method $\mathrm{B}$. This iterative process of beam elimination and new beam computation stops when user SIRs are acceptable. We measure average subcarrier throughput, namely average number of assigned users per subcarrier. In figure 2, this is illustrated as a function of number of transceivers for $M=4$ antennas, for different multi-path (number of paths, $L$ ) and $\gamma=10 \mathrm{~dB}$. We see that for the same multi-path conditions, approach $\mathrm{B}$ always performs better than $\mathrm{A}$ due to the iterative beam computations as opposed to $\mathrm{A}$, where beams are computed once. Moreover, different criteria for computation of new beam were used in A 
and B. For relatively small $C$, approach B outperforms A by $20 \%$, while for larger $C, \mathrm{~B}$ is better than A by $4 \%$. Note that the throughput with $L=2$ is alaways larger than that for $L=1$ due to the additive effect of multi-path.

The most significant observation is that performance improves as $C$ increases until $C$ reaches a limit value $C^{*}$, beyond which no further improvement occurs. Then, the system has reached spatial separability performance limits and cannot accommodate more users in the same subcarriers. Thus, for A and B and $L=1$, we have $C_{A}^{*}=17$ and $C_{B}^{*}=13$ with corresponding throughput of 1.9 and 2.18 users per subcarrier. For $L=2$, it is $C_{A}^{*}=12$ and $C_{B}^{*}=9$ with throughput of 2.4 and 2.5. It was also observed that the benefit of $\mathrm{B}$ over $\mathrm{A}$ increases as $M$ increases and decreases as $L$ increases. Furthermore, the number $C^{*}$ is almost proportional to $M$. Although in a realistic system $N$ and $K$ are larger, performance is still determined by subcarrier reuse, which in turn depends on spatial and multi-path channel properties of users, beamforming and resource allocation policy. For a given BER requirement and a given value of $M$, there exists a crucial number of transceivers $C^{*}$, beyond which no further performance benefits are anticipated. Viewed differently, $C$ can be made as small as $C^{*}$ at the design stage with no incurred performance losses.

\section{Discussion}

In this study, we identified the particular features of the coupled resource allocation problem and demonstrated the impact of smart antenna systems with transceiver limitations on the design of MAC layer algorithms. There exist several directions for future study. A first issue is establishing a theoretical framework for determining the limiting system performance. A more general treatment could include power adaptation. Finally, the analogy between the addressed assignment problem and the scheduling problem at the packet level (where the transceiver is viewed as a server) is worth considering. The arising issue is the identification of eligible user activation sets for each subcarrier by considering spatial separability, number of subcarriers per user and queue lengths. Several existing ideas and stabilizing scheduling policies could be extended to such generalized scheduling problems.

\section{References}

1. K. Sheikh, D. Gesbert, D. Gore and A. Paulraj, "Smart antennas for broadband wireless access networks", IEEE Commun. Mag., vol.37, no.11, pp.100-105, Nov. 1999.

2. T. Keller and L. Hanzo, "Adaptive multicarrier modulation: a convenient framework for time-frequency processing in wireless communications", Proc. IEEE, vol.88, no.5, pp.611-640, May 2000.

3. F. Rashid-Farrokhi, K.J.R. Liu and L. Tassiulas, "Transmit beamforming and power control for cellular wireless systems", IEEE J. Select. Areas Commun., vol.16, no.8, pp.1437-1450, Oct. 1998. 
4. C. Farsakh and J.A. Nossek, "Spatial Covariance based downlink beamforming in an SDMA mobile radio system", IEEE Trans. Commun., vol.46, no.11, pp.1497-1506, Nov. 1998.

5. F. Shad, T.D. Todd, V. Kezys and J. Litva, "Dynamic slot allocation (DSA) in indoor SDMA/TDMA using a smart antenna basestation", IEEE/ACM Trans. Networking, vol.9, no.1, Feb. 2001.

6. K.-W. Wong, R.S.-K. Cheng, K. Ben Letaief and R.D. Murch, "Adaptive antennas at the mobile and base stations in an OFDM/TDMA system", IEEE Trans. Commun., vol.49, no.1, pp.195-206, Jan. 2001.

7. I. Koutsopoulos and L. Tassiulas, "Adaptive resource allocation in wireless broadband networks with OFDM signaling", Proc. IEEE INFOCOM 2002 vol.3, pp.13761385.

8. I. Koutsopoulos, "Resource Allocation issues in wireless broadband networks with OFDM signaling", Ph.D Dissertation, University of Maryland, College Park, December 2002 . 